\title{
Grain Size Measurement of Crystalline Products Using Maximum Difference Method
}

\author{
Leena Lepistö ${ }^{1}$, Iivari Kunttu ${ }^{1}$, Matti Lähdeniemi ${ }^{1}$, Tero Tähti ${ }^{2}$, and Juha Nurmi ${ }^{2}$ \\ ${ }^{1}$ Satakunta University of Applied Sciences \\ Faculty of Technology and Maritime Management \\ Tekniikantie 2, FI-28600 Pori, Finland \\ \{Leena.Lepisto, Iivari.Kunttu\}@samk.fi \\ http: / / www. samk.fi \\ ${ }^{2}$ Danisco Texturants \& Sweeteners, Innovation \& Technology, \\ Sokeritehtaantie 20, FI-02460 Kantvik, Finland \\ \{Tero.Tahti, Juha.Nurmi\}@danisco.com \\ http: //www.danisco.com
}

\begin{abstract}
Texture analysis methods are widely used in various monitoring and measurement tasks in machine vision solutions. In this paper we present a novel method for the determination of grain size distributions in the manufacturing processes of crystalline products. Our method, maximum difference histogram $(\mathrm{MDH})$, is based on statistical gray level differences in the texture images. Using this method, it is possible to estimate the grain size distributions in the images. It is also possible to monitor the average grain sizes in the image series acquired during the crystallization process. This is carried out by determining the center of gravity $(\mathrm{CoG})$ of the distribution represented by MDH. Experimental results obtained from images acquired from a carbohydrate crystallization process reveal that the proposed method is useful in in-line grain size measurement tasks.
\end{abstract}

\section{Introduction}

The use of image information in material characterization has been in strong growth during recent years. Materials in various industrial processes can be effectively characterized and their visual properties can be measured using image analysis and machine vision methods. In the process industry, significant amounts of information on the process can be acquired using machine vision. This information is utilized in process monitoring and control tasks.

The inspection and measurements of granular products is a fundamental problem in several industrial processes. Traditionally, the characterization of the granular products has been carried out by manual inspection. That is, the grain properties have been inspected by sieving or microscope analysis. This kind of approach is time consuming and hence unable to provide on-line information from the granulation process. For this reason, image analysis methods have been adopted to inspection of grain properties in several industrial processes. In the field of pharmaceutical sciences, the characterization of grain size distributions of powders has become an actively studied topic. Laitinen et al. [2],[3] for example, have used image analysis methods for the 
inspection of pharmaceutical powder distributions. In the field of crystallization, $\mathrm{Qu}$ et al. [7] have inspected crystal growth using image analysis applied to image material acquired using a video microscope.

The application field of this paper is related to the manufacturing process of crystalline products. In this process the average size and size distribution of the product play a constitutive role. The properties of the crystal population are important as well for the end use functionality as for the ease of downstream processing. In industrial processes fluctuations in the processing conditions can occur for several reasons. Such fluctuations must be compensated by the operators in order to keep the product quality stable. Visual observation of the process has remained an important tool in evaluation of the expected product quality in terms of crystal size. Up to now quantitative size measurements from the in-line process data have been scarce due to difficulties in receiving reliable information on the crystal size from very dense suspensions present in industrial processes.

Texture analysis is common in various industrial machine vision applications. Texture analysis is used to e.g. estimate different properties of surfaces. Typical industrial applications of texture analysis include the inspection of different surface materials such as paper, metal or textiles [6]. In these applications, the classification and recognition of different surfaces is based on texture properties. These properties are for example roughness, granular size or directionality of texture. According to Rao and Lohse [8], the most essential texture properties in human perception are repetitiveness, directionality, granularity, and complexity. The directionality of nonhomogenous natural textures has been discussed in our earlier work [4]. The analysis of grain properties in rock images can be found in [5].

When image analysis is utilized in the analysis of grain properties of crystalline products, one needs to choose between two kinds of approaches. The traditional approach is to extract the grains from the image by using some image segmentation method. The grain properties, such as size or shape, are then measured from the segmented image. However, there are several difficulties with this kind of approach. Firstly, the image segmentation algorithms are often sensitive to illumination changes in the process. In addition, the color (or gray level) distributions of the grains are not always homogenous. On the contrary, the colors of the grains to be extracted from the images may vary significantly. These factors may cause difficulties in the segmentation process. Secondly, the segmentation causes computational load that may be critical in the case of on-line analysis and inspection applications. An alternative for the extraction of the grains from the image background is the employment of texture analysis in the granularity measurements. It has been found that texture analysis methods can be applied to the analysis and inspection of granular products. Using texture analysis tools, it is often advantageous to inspect the particle populations as larger surfaces, not by extracting single grains from the image. Compared to the traditional approach, texture analysis does not have the problems related to image segmentation process. In addition, the whole image can be used in the granularity inspection, not only the single grains.

In this paper, we apply texture analysis methods for granularity analysis of crystalline products. The testing material used in this study is image data obtained from the 
crystallization process in carbohydrate manufacturing. The rest of this paper is organized as follows. Chapter 2 describes statistical texture analysis methods and particularly the proposed method, Maximum difference histogram. In Chapter 3, we present two kinds of experiments in which the proposed texture analysis method is used to estimate granular sizes of the carbohydrate crystals. The experimental results are discussed in Chapter 4.

\section{Statistical Texture Analysis}

Numerous techniques have been proposed for texture description. Tüceryan and Jain [9] have divided texture description methods into four main categories: statistical, geometric, model-based, and signal processing methods. In this study, we concentrate on the statistical texture analysis methods.

Statistical techniques are based on the description of the spatial organization of the image grey levels. On the basis of the grey level distribution, it is possible to calculate several kinds of simple statistical features. Grey level co-occurrence matrix developed by Haralick [1] has been a popular tool in texture analysis and classification. Cooccurrence matrix estimates the second order joint probability density functions $g(i, j \mid$ $d, \Theta)$. Each $g(i, j \mid d, \Theta)$ is the probability of going from grey level $i$ to grey level $j$, when the relative position between the gray levels is $d$ and the direction is $\Theta$. These probabilities create the co-occurrence matrix $\mathbf{M}(i, j \mid d, \Theta)$. It is possible to extract textural features from the matrix [1]. The most commonly used textural features extracted from the matrix are contrast, entropy, and energy. Preliminary experiments have revealed the contrast is the most sensitive of these features for granularity.

In addition to the co-occurrence matrix, several other statistical texture analysis methods have been presented. One example of them is grey level difference method originally presented by Weszka et al. [10]. In this method, the histograms formed by absolute differences between pairs of gray levels are used. The grey level difference calculation is based on pixel pairs whose relative position is defined by displacement vector $\boldsymbol{d}=\left(d_{x}, d_{y}\right)$.

\subsection{Maximum Difference Method}

In the statistical methods presented in the previous Chapter, the relative position or distance between the gray level pairs, $d$, is fixed. Consequently it is necessary to define constant spatial distance between the gray levels used in the statistical measurement for the whole image set to be inspected. This can be problematic because in certain texture types the grain sizes are varying. For this reason, the use of fixed distance $d$ is not practical, especially in the cases in which texture analysis is employed to measure the granular sizes of texture. For this reason, fixed distance is not the best alternative for granularity measurements.

In our approach, we use adaptive relative position $d$ in the texture statistics. In the proposed statistical texture measure, maximum difference histogram, a histogram formed by relative positions that produce maximum difference between gray levels of the pixel pairs is formed. The method can be presented as follows. 
1. The texture image is inspected pixel by pixel.

2. For each pixel (gray level) $i$, select the following $n$ pixels in direction $\Theta$.

3 . Find the highest absolute difference between gray level $i$ and the gray levels within the following $n$ pixels. If computational efficiency is required, the $n$ should be selected small.

4. The gray level $j$ that has the highest absolute difference to gray level $i$ is selected.

5. The spatial distance $d$ between gray levels $i$ and $j$ is determined.

6. This procedure is repeated for each gray level of the texture. The values of $d$ are presented as a histogram.

The resulting histogram is called maximum difference histogram (MDH), and it can be employed in the estimation of the grain size distribution. If only the average of the grain sizes in the population is interesting, it is possible to determine it based on the histogram. This can be done by determining the center of gravity $(\mathrm{CoG})$ of the histogram.

\section{Experiments}

In the experimental part of this paper, we present two kinds of test results obtained from the granularity inspection of carbohydrate crystals. In the first one, we use the proposed maximum difference histogram method for the estimation of granular size distributions of images acquired from the carbohydrate crystallization process. In the second experiment, an in-line measurement of the crystallization process is presented. Before these experiments, is given a short description of the carbohydrate manufacturing process.

\subsection{Carbohydrate Manufacturing Process}

The crystallizing substance used in this study is a carbohydrate product. The crystallization process of the carbohydrate is based on evaporation of the solvent in a constant temperature. The resulting supersaturation in the carbohydrate-solution acts as a driving force for the phase change operation from liquid to solid. During the evaporation process the final crystal population is created through growth of existing crystals and formation of new crystals, starting from an initially given seed crystal population. The target is to obtain a pre-defined average crystal size and a possibly narrow crystal size distribution. This is achieved by balancing the processes of crystal growth and crystal formation by adjustment of the process parameters.

\subsection{Determination of Grain Size Distribution}

In the first experiment, we estimated grain size distributions of some selected microscope images obtained from the carbohydrate crystallization process. We used the maximum difference method to make a histogram that estimates the grain size distributions of the images. Figure 1 presents two examples of the grain images and their maximum difference histograms. The purpose is to estimate the grain size distribution. The histograms express normalized granular size distributions of the images in pixels. In all the histograms, the distance $d$ is determined in horizontal direction. 
a)

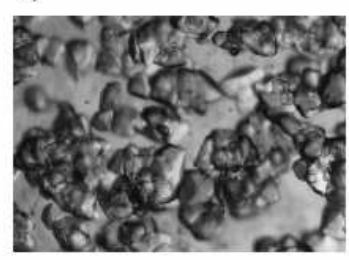

b)

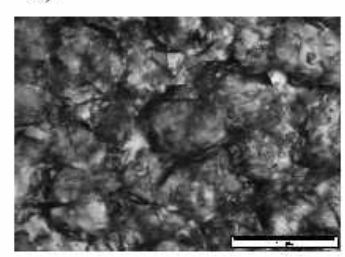

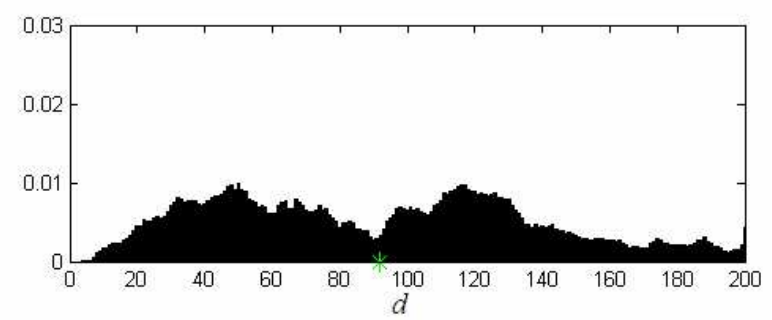

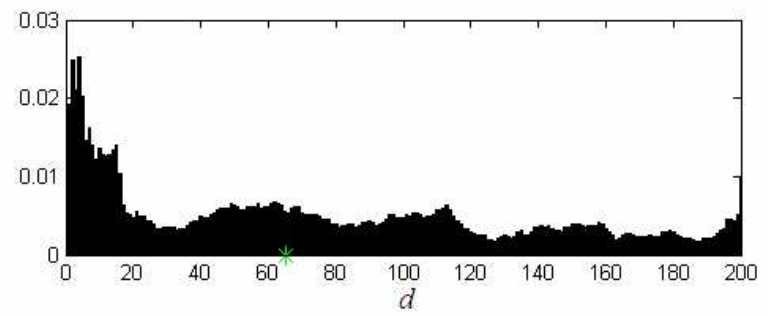

Fig. 1. Grain images and their respective maximum difference histograms. The center of gravity $(\mathrm{CoG})$ is marked with green star.

The results presented in figure 1 show that the Maximum difference histogram is able to estimate the grain size distributions with reasonable accuracy. In subfigure (b), the effect of small grains is visible in the histogram. On the other hand, in subfigure (a), these small particles are not present. So, the method can be used to track the formation of small crystals during the process. However, just by looking at the obtained average value the growth into larger crystals visible in subfigure (b) can not be detected. The histograms shown in figure 1 represent number based distributions of the crystal sizes. If the small particles are not considered interesting, the growth of larger crystals can be followed easier by representing the histograms in a volume based $\left(d^{3}\right)$ form. The grain image presented in subfigure (a) has also certain smooth regions which do not contain any grains. These kinds of regions can be problematic for the proposed grain size estimation method and they may cause some errors to the distributions. Also noise and other distortions may cause small variations. However, the method seems to estimate the mean grain size (marked with green star in figure 1) sufficiently good. Another topic to be considered is the transform from pixels to metric system, because distribution expresses the grain sizes in pixels. This can be done experimentally.

\subsection{Measurement of Mean Grain Level in the Crystallization Process}

In the practical carbohydrate manufacturing, one needs to monitor and control the growth of the grain size during the crystallization process. In the beginning of the process, the grains are small sized and their amount is also small. However, the grains start growing very soon after the beginning of the process and also their number grows fast. After that, the grain sizes tend to stabilize on certain level although their shapes get smoother. To monitor the grain size growth, one needs some image descriptor that is capable of expressing the mean grain size in each image. 
12

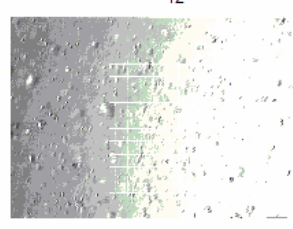

57

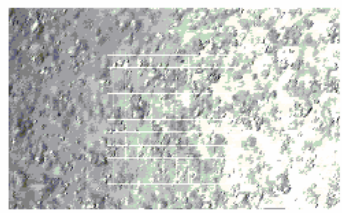

296

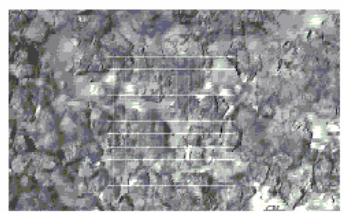

30

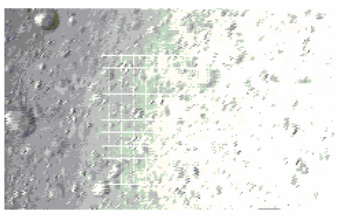

86

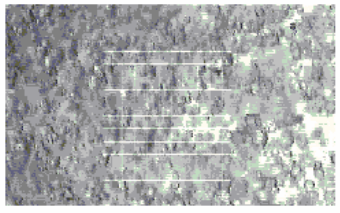

851

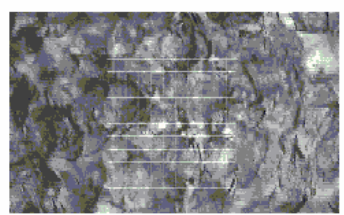

40

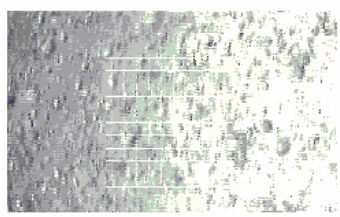

173

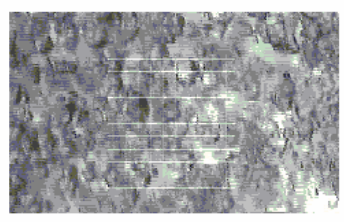

1112

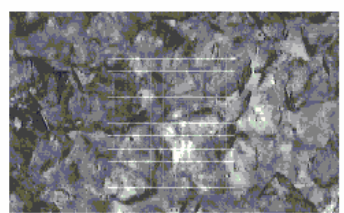

Fig. 2. Example images from the test set acquired from the carbohydrate crystallization process. The images are numbered from 1 to 1200 .

In order to test the procedure for monitoring an industrial crystallization process using textural methods, images were acquired through a common process control microscope in a real production process. Images were taken using a process microscope and a process surveillance camera attached on the side of the evaporation vessel. The camera was connected to a computer with a frame grabber card. Images were saved on computer using Image-Pro Plus image analysis software. Additional inhouse software was used for automation and timing of the image capture procedure. The image acquisition was started at the time of seed crystal addition and stopped at the end of the process. That means that in addition to crystal size and crystal size distribution, also the solids fraction in the images changes remarkably. The resulting set of images contained approximately 1200 images. Image size was $768 \times 460$ pixels. Figure 2 shows some example images acquired in different phases of the crystallization process.

We used maximum difference method to estimate the growth of the grain size. In this experiment, only the average size of the grains was determined. This was carried out by calculating the center of gravity $(\mathrm{CoG})$ for each $\mathrm{MDH}$. The MDH was calculated using value 50 for parameter $n$ in horizontal direction. The CoG was calculated for each image in the sequence. Figure 3 presents the $\mathrm{CoG}$ during the process.

The results presented in figure 3 show that the graph representing the center of gravity follows the real crystal growth process. The graph rises very fast between samples 1 and 150. After that, it stabilizes on certain level. This kind of growth can be seen also in the image set presented in figure 2. Based on manual inspection, this kind 


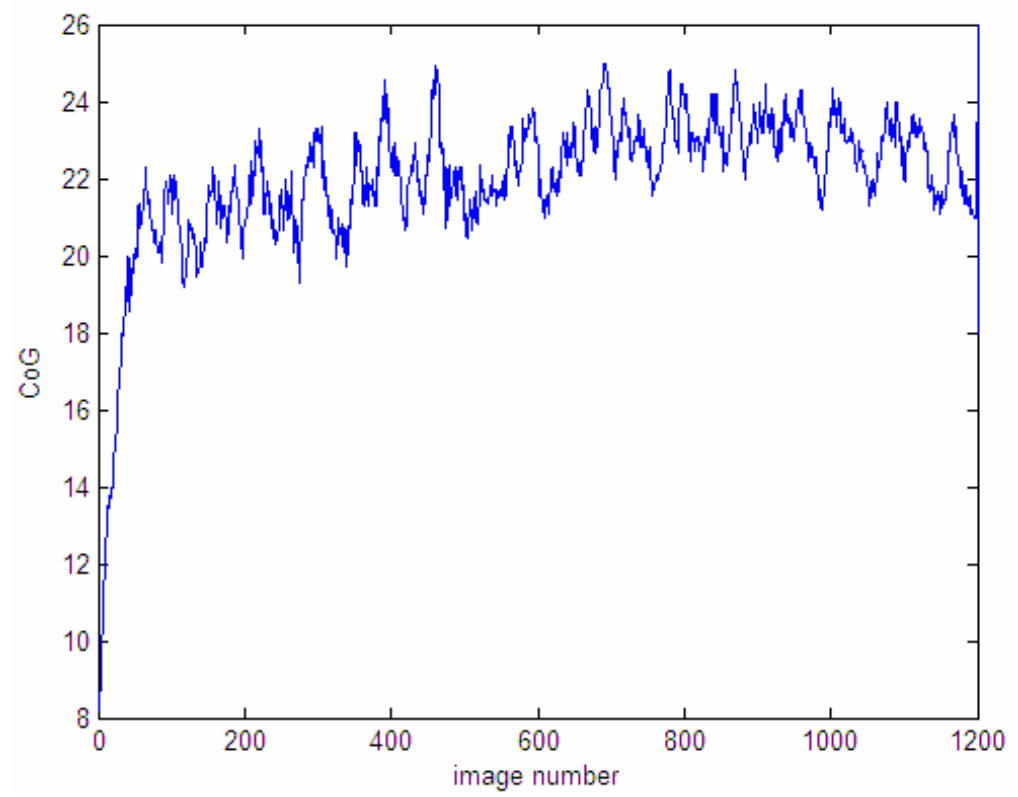

Fig. 3. Mean grain sizes in the sequence of 1200 crystal images acquired from a carbohydrate crystallization process

of graph seems to estimate the real development in the crystal population during the process reasonably well. Formation of new crystals and attrition of existing large crystals sets into a dynamic balance, while the number of crystals keeps rising until the end of the process.

\section{Discussion}

In this paper we have presented a novel texture description method, Maximum difference histogram for the estimation of grain size distributions in the images. The experimental results reveal that this method is able to relatively well estimate the mean grain size in the population. The distribution is somewhat sensitive to noise and other distortions in the image. Also areas without grains may cause problems. For this reason, the shape of the distribution may have small differences. These difficulties can be overcome by using e.g. image preprocessing, edge detection and thresholding, or other image enhancement before the calculation of the histogram. This could be subject of further investigations.

On the other hand, in its current form the Maximum difference histogram method has proved to be able to approximate the mean grain size in the grain population. We employed the method in the monitoring of carbohydrate production. The experimental results show that the method is able to estimate the grain growth in the crystallization process.

The computational cost of the proposed method is at the same level as with other statistical texture analysis methods. The computation can be made lighter by e.g. 
limiting the inspection area in the pixel neighborhoods (selecting parameter $n$ ). In any case, the computational cost is not a significant problem in the use of the proposed method in in-line process monitoring.

In conclusion, the Maximum difference histogram method is able to estimate the mean grain sizes of a particle distribution relatively accurately. It is suitable to be used in real process monitoring tasks in industrial machine vision applications.

\section{Acknowledgments}

The authors wish to thank Danisco Sweeteners \& Pharma for assistance with the image acquisition in the plant, and Mr. Esa Wainio from Picomega Oy for invaluable help with the image acquisition tools.

\section{References}

1. Haralick, R.M., Shanmugam, K., Dinstein, L.: Textural Features for Image Classification. IEEE Transactions on Systems, Man, and Cybernetics 3, 610-621 (1973)

2. Laitinen, N., Antikainen, O., Yliruusi, J.: Does a powder surface contain all necessary information for particle size distribution analysis? European Journal of Pharmaceutical Sciences 17, 217-227 (2002)

3. Laitinen, N., Rantanen, J., Antikainen, O., Yliruusi, J.: New perspectives for visual characterization of pharmaceutical solids. Journal of Pharmaceutical Sciences 93, 165-176 (2004)

4. Lepistö, L., Kunttu, I., Autio, J., Visa, A.: Classification Method for Colored Natural Textures Using Gabor Filtering. In: Proceedings of 12th International Conference on Image Analysis and Processing, pp. 397-401 (2003)

5. Lepistö, L., Kunttu, I., Autio, J., Visa, A.: Rock image retrieval and classification based on granularity. In: Proceedings of 5th International Workshop on Image Analysis for Multimedia Interactive Services (2004)

6. Pietikäinen, M., Ojala, T., Silven, O.: Approaches to texture-based classification, segmentation and surface inspection. In: Chen, C.H., Pau, L.F., Wang, P.S.P. (eds.) Handbook of Pattern Recognition and Computer Vision, 2nd edn. pp. 711-736. World Scientific Publishing Company, Singapore (1998)

7. Qu, H., Louhi-Kultanen, M., Kallas, J.: In-line image analysis on the effects of additives in batch cooling crystallization. Journal of Crystal Growth 289, 286-294 (2006)

8. Rao, A.R., Lohse, G.L.: Towards a texture naming system: identifying relevant dimensions of texture. In: Proceedings of IEEE Conference on Visualization, San Jose, California, pp. 270-227 (1993)

9. Tüceryan, M., Jain, A.K.: Texture Analysis. In: Chen, C.H., Pau, L.F., Wang, P.S.P. (eds.) Handbook of Pattern Recognition and Computer Vision, pp. 235-276. World Scientific, Singapore (1993)

10. Weszka, J.S., Dyer, C.R., Rosenfeld, A.: A Comparative Study of Texture Measures for Terrain Classification. IEEE Transactions on Systems, Man, and Cybernetics 6, 269-285 (1976) 